\title{
Obstructive sleep apnea: Screenings in primary, secondary and tertiary care settings
}

\author{
Atmaram Yarlagadda ${ }^{1}$, Jayaprada Kasaraneni ${ }^{2}$, Anita H. Clayton ${ }^{3^{*}}$ \\ ${ }^{1}$ McDonald Army Health Center, Ft. Eustis, USA; atma@golfolks.com \\ ${ }^{2}$ Research Assistant Vaccine and Gene Therapy OHSU, Portland, USA; kasaranenij@gmail.com \\ ${ }^{3}$ Department of Psychiatry \& Neurobehavioral Sciences, University of Virginia, Charlottesville, USA; \\ *Corresponding Author: ahc8v@virginia.edu
}

Received 19 June 2013; revised 20 July 2013; accepted 10 August 2013

Copyright (C) 2013 Atmaram Yarlagadda et al. This is an open access article distributed under the Creative Commons Attribution License, which permits unrestricted use, distribution, and reproduction in any medium, provided the original work is properly cited.

\begin{abstract}
Insomnia is a major health problem affecting millions of people around the globe, particularly in the developed world, substantially impacting on individual work performance and raising disability costs. Insomnias can be broadly divided into primary and secondary types. Primary insomnias can further be divided into parasomnias and dyssomnias. Diagnostic approaches to insomnias can be challenging, thereby complicating the treatment process. Obstructive sleep apnea (OSA) falls under the category of primary insomnias and is a good example of frequently missed diagnoses. The goal of this paper is to document the development of a self-reporting sleep apnea scale based on graded percentage scores to correlate with primary, secondary and tertiary risks. Scores falling under fifty percent represent primary prevention risks of OSA and suggest possible interventions. A score of fifty percent highlights three core symptoms on the scale as described in detail below to represent early detection of OSA or secondary prevention. Finally, scores exceeding fifty percent will include associated risks of co-morbidities as well as other factors contributing to OSA, or tertiary prevention, with separate scores assigned to individual risk factors. Analysis of final scores on the proposed scale would therefore, in our opinion help achieve the goal of grading the risk of OSA from a multidisciplinary preventive care aspect.
\end{abstract}

Keywords: Sleep Apnea; Primary Prevention; Insomnia; Obesity; Metabolic Syndrome

\section{INTRODUCTION}

Obstructive sleep apnea (OSA) is a breathing related sleep disorder categorized under dyssomnias. OSA is very common and often thought to be closely linked to age, male sex and obesity [1,2]. However, this is a misconception as it can be unrelated to age and obesity with an increasing prevalence in younger and non-obese individuals $[3,4]$ or the "atypical patient". Awareness of this condition is low, and OSA frequently goes unrecognized in various health care settings including medical providers, health maintenance organizations/centers, hospitals and nursing homes [5]. Morbidity and mortality rates related to OSA, estimated by both direct and indirect costs are staggering due to under diagnosis of the condition at multiple levels of preventive care [6,7]. In this article, we will highlight the increasing prevalence of OSA [8] by introducing a simple self-report screening tool to rule out OSA at all levels of primary, secondary and tertiary preventive care. Increasing OSA awareness in the extended medical community, as well as in the average person, can promote better clinical outcomes and overall improved quality of life.

\section{PRIMARY CARE}

Characteristics of insomnias in primary care settings can be varied and clinically challenging when broken down by symptomatology i.e., difficulty initiating sleep (DIS), difficulty maintaining sleep (DMS), early morning awakenings (EMA) and nonrestorative sleep (NRS). DMS, however, tops the list as the most commonly reported insomnia symptom at $80.25 \%$ according to a recent international study conducted in 5293 patients, EQUINOX (Evaluation of daytime Quality Impairment by Nocturnal awakenings in Outpatient's experience) [9]. 
The study also reports a significant link between DMS, EMA, NRS and other insomnia symptoms. An estimated $10 \%$ of the adult population suffers from chronic insomnia in whom primary insomnia comprises $25 \%$ [10]. OSA appears to affect between $2 \%-4 \%$ of middle-aged women and men making up for a large proportion of primary insomnias and potentially accounting for many sleep complaints in adults in primary care settings [11]. Obesity and increasing age also play significant roles in the increasing prevalence of OSA [12]. The rise in body mass index (BMI) and medical comorbidities are shown to be directly associated with both the prevalence and the severity of OSA [13]. Obesity is a growing global problem associated with serious morbidity and mortality with sleep disturbances at the core of comorbid pathology [14]. Evidence from the literature relating to childhood obesity (with a prevalence of $30 \%$ in adolescents) and growing concerns of pediatric sleep apnea in older children and adolescents mandates a closer look at the disorder from a primary prevention aspect [15].

A healthy lifestyle with regular exercise associated with weight loss has been shown to reverse or improve OSA in selected patients [16]. On the other hand, obesity, aging and snoring do not represent necessary diagnostic criteria for OSA, and reliance on these symptoms may lead to failure to assess for OSA in non-obese, young individuals who may still constitute an "at risk/atypical" population [4]. Therefore, it is important to consider symptoms such as daytime sleepiness, fatigue, depression, lack of sexual desire and headaches in the diagnosis of OSA regardless of associated conditions like obesity, snoring, and advanced age. Characteristics/type of insomnia, duration of insomnia, level of occupational and social impairment, along with headaches, depression, anxiety and sexual dysfunction in an individual should prompt the primary care provider towards screening and early detection of OSA [17]. Appropriate diagnosis of OSA is also of immense public health concern in all aspects of preventive health care [18]. Observational studies indicate reduction of motor vehicle crashes among drivers with OSA when diagnosed early and treated with continuous positive airway pressure (CPAP) [19-22]. Failed primary preventive care measures (risk reduction) and low awareness (lack of education) could ultimately translate into higher incidence and prevalence of unrecognized and untreated OSA.

\section{SECONDARY CARE}

Early detection, or the identification of new cases (incidence) should be emphasized as OSA is frequently under-recognized, thus, delaying interventions that mitigate complications. Although not absolute, obesity comprises a huge risk factor and BMI is an important benchmark for OSA risk [23]. One such example is the increasing prevalence of pediatric sleep apnea associated with the rising epidemic of childhood obesity [15]. Frequently, overweight children present with breathing problems that impact sleep which may translate into behavioral issues during the daytime (hyperactivity). These behavioral problems (hyperactivity, restlessness, disruptive behaviors, poor concentration and attention), may be commonly labeled as attention deficit disorders in children who may receive stimulants due to unrecognized OSA [24-26]. Another potential site for early detection of OSA would be ear, nose, and throat clinics where nasal septal deviation, enlarged tonsils/adenoids, and craniofacial abnormalities may be considered breathing problems as opposed to breathing-related sleep problems [27]. Lack of proper screening for breathing-related sleep disorders in allergy, immunology and pulmonary clinics, especially in children, could lead to increased complications secondary to decreased immunity resulting from hypoxemia and insomnia due to OSA [28]. Prevalence of metabolic syndrome in association with obesity continues to rank high in almost all clinical settings drawing more attention to primary preventive measures [29]. Screening for sleep disorders, especially OSA, in patients with cardiac risk factors associated with myocardial infarction and neurological risk factors leading to strokes is uncommon [30]. The current obstetric literature points to the growing risks of maternal and neonatal morbidities associated with OSA complicating pregnancy [31]. This risk is amplified by pre-existing maternal obesity and hypertension leading to pre-eclampsia and preterm birth. Early detection and use of CPAP is thought to significantly decrease these complications. In psychiatry, symptoms such as insomnia and depression are well known to have a bidirectional association or relationship [32]. We have in the past described the link between OSA and depression, which is frequently overlooked due to lack of proper sleep assessment [33]. Most psychiatric medications (antidepressants, mood stabilizers, antipsychotics, sedatives and anxiolytics) carry the risk of weight gain and metabolic syndrome making a strong case for pre-screening of breathing-related sleep disorders mandatory [34] under those circumstances. Early detection and management of OSA could therefore decrease the prevalence rate by decreasing tertiary care complications related to preexisting pathology.

\section{TERTIARY CARE}

Increased prevalence of OSA remains a problem in tertiary care medicine as described above and can masquerade as complications of pre-existing disease entities, with progression of the underlying disease process. Examples of specialties in medicine that should increase 
screening for moderate-to-severe OSA are: 1) Anesthesiology, to decrease the risks of peri-operative adverse events [35]; 2) Cardiology, to prevent acute ischemic events and repeated myocardial infarctions [36,37]; 3) Neurology, to prevent strokes in patients suffering from high blood pressure [38]; 4) Pulmonary, to improve ventilation in COPD and other chronic lung diseases [39]; 5) Obstetrics, to prevent complications associated with obesity during pregnancy (C-section, pre-eclampsia) and hypoxemic fetal complications i.e., low birth weight by improving maternal health and overall outcomes [40]; 6) Geriatrics, to improve quality of life in elderly patients especially those suffering from dementia due to vascular or other chronic conditions where hypoxemia due to OSA can have a significant impact [41]; and 7) Psychiatry, to decrease comorbidities associated with weight gain and metabolic syndrome [42].

\section{DISCUSSION}

Timely recognition and diagnosis of OSA therefore, is an essential aspect of preventive medicine. The proposed sleep apnea scale (attached) is designed to cover all settings of preventive care with distribution of scores related to the clinical significance of symptoms. For example, scores under fifty percent may represent risks falling under primary prevention care and can be monitored longitudinally. Scores at fifty percent on the other hand, are indicative of early diagnosis of OSA and fall under secondary prevention and are represented by three core symptoms on the scale with scores assigned in the following manner; question \#1 difficulty maintaining sleep rated at ten percent, question \#2 occupational and social impairment secondary to insomnia also rated at ten percent and question \#3 snoring with observed choking rated at thirty percent, contributing to fifty percent of the total score on the scale. The need for confirmation with polysomnography (PSG) in this scenario becomes crucial.

Scores higher than fifty percent indicate presence of OSA and complications associated with it seen commonly in tertiary care prevention. An example would be physical attributes seen in question \#7 such as increased BMI, which is rated twenty percent on the scale. The addition of three core symptoms to question \#7 raises the combined score to seventy percent increasing the probability of the presence of OSA related complications in a given individual.

Individuals who lack physical attributes (no increase in BMI), but respond "yes" to the question \#5 representing sleep deprivation, fatigue and increased daytime sleepiness score ten percent on the scale. In combination with three core symptoms required for diagnosis of OSA, this would amount to a combined risk of sixty percent on the scale. Comorbidities and the number of prescription medications are each individually assigned a score of five percent (questions $\# 4, \# 6$, \#8, and \#9) contributing to the increased risk of OSA related complications. By checking "yes" to additional question(s) beyond the core three symptoms which comprise OSA, the "atypical but still at risk" population can potentially be detected with increasing sensitivity on the scale. Other significant risk factors such as gender and age are included in the scale but have not been assigned scores. Male gender and advancing age are considered high risk factors for OSA. However, risk factors for OSA can widely vary within a sub-group of individuals. For example, the risk increases in women during pregnancy, in children with moderate-severe obesity, in adults with coronary artery disease, stroke and dementia, in asthmatics with chronic pulmonary complications, and in psychiatric patients receiving psychotropic medications for mood or thought disorder. Screening for OSA and appropriate interventions not accomplished promptly may worsen the risk e.g. death during general anesthesia in people undergoing surgery.

\section{CONCLUSION}

Evidence based outcomes demonstrating the benefits of early or timely screening for OSA is currently unavailable to support the extended use of a graded selfreport sleep apnea scale [42]. Therefore, validation studies to incorporate such a scale for grading the risks for primary, secondary and tertiary care interventions in an outpatient setting are important. Validation and subsequent implementation of such a screening tool would likely have positive outcomes, and may also be extended into non-clinical areas to improve public safety by raising awareness of OSA.

\section{REFERENCES}

[1] Kryger, M.H. (2000) Diagnosis and management of sleep apnea syndrome. Clinical Cornerstone, 2, 39-47. doi:10.1016/S1098-3597(00)90039-5

[2] Young, T., Evans, I., Finn, I. and Plta, M. (1997) Estimation of the clinically diagnosed proportion of sleep apnea syndrome in middle aged men and women. Sleep, 20, 705-706.

[3] O'Keeffe, T. and Patterson, E.J. (2004) Evidence supporting routine polysomnography before bariatric surgery. Obesity Surgery, 14, 23-26. doi: $10.1381 / 096089204772787248$

[4] Lettieri, C.J., Eliasson, A.H., Andrada, T., Khramtsov, A., Raphaelson, M. and Kristo, D.A. (2005) Obstructive sleep apnea syndrome: Are we missing an at-risk population? Journal of Clinical Sleep Medicine, 1, 381-385.

[5] Kramer, N., Cook, T.E., Carlisle, C.C., Corwin, R.W. and Millman, R.P. (1999) The role of primary care physician in recognizing obstructive sleep apnea. Archives of Inter- 
nal Medicine, 159, 965-968. doi:10.1001/archinte.159.9.965

[6] Turkington, P.M., Sircar, M., Allgar, V. and Elliott, M.W. (2001) Relationship between obstructive sleep apnea, driving simulator performance, and risk of road traffic accidents, Thorax, 56, 800-805.

doi:10.1136/thorax.56.10.800

[7] Shahar, E., Whitney, C.W., Redline, S., Lee, E.T., Newman, A.B., Javler, F., George, N., O'Connor, T., Boland, L.L., Schwartz, J.E. and Samet, J.M. (2001) Sleep-disordered breathing and cardiovascular disease: Cross-sectional results of the sleep heart health study. American Journal of Respiratory and Critical Care Medicine, 163, 19-25. doi:10.1164/ajrccm.163.1.2001008

[8] Netzer, N.C., Hoegel, J.J., Loube, D., et al. (2003) Prevelence of symptoms and risk of sleep apnea in primary care. Chest, 124, 1406-1414.

doi:10.1378/chest.124.4.1406

[9] Leger, D., Partinen, M., Hirshkowitz, M., Chokroverty, S., Hedner, J. and EQUINOX (Evaluation of daytime Quality Impairment by Nocturnal awakenings in Outpatient's eXperience) (2010) Characteristics of insomnia in a primary care setting: EQUINOX survey of 5293 insomniacs from 10 countries. Sleep Medicine, 11, 987-998. doi:10.1016/j.sleep.2010.04.019

[10] Roth, T. and Roehrs, T. (2003) Insomnia: Epidemiology, characteristics, and consequences. Clinical Cornerstone, 5, 5-15. doi:10.1016/S1098-3597(03)90031-7

[11] Young, T., Palta, M., Dempsey, J., Skatrud, J., Weber, S. and Badr, S. (1993) The occurrence of sleep disordered breathing among middle-aged adults. The New England Journal of Medicine, 328, 1230-1235. doi:10.1056/NEJM199304293281704

[12] Kapur, V., Strohl, K.P., Redline, S., Iber, C., O’Connor, C. and Neito, J.L. (2002) Underdiagnosis of sleep apnea syndrome in United States communities. Sleep Breath, 6, 49-54. doi:10.1055/s-2002-32318

[13] Neito, F.J., Young, T.B., Lind, B.K., et al. (2000) Association of sleep-disordered breathing, sleep apnea, and hypertension in a large community-based study. Sleep Heart Health Study. JAMA, 283, 1829-1836. doi:10.1001/jama.283.14.1829

[14] Gami, A.S., Caples, S.M. and Somers, V.K. (20030 Obesity and obstructive sleep apnea. Endocrinology and Metabolism Clinics of North America, 32, 869-894. doi:10.1016/S0889-8529(03)00069-0

[15] Levers-Landis, C.E. and Redline, S. (2007) Pediatric sleep apnea: Implications of the epidemic of childhood overweight. American Journal of Respiratory and Critical Care Medicine, 176, 1054-1055.

[16] Kajaste, S., Brander, P.E., Telakivi, T., Partinen, M. and Mustajoki, P. (2004) A cognitive-behavioral weight reduction program in the treatment of obstructive sleep apnea syndrome with or without initial nasal CPAP: A randomized study. Sleep Medicine, 5, 125-131. doi:10.1016/j.sleep.2003.07.007

[17] Dement, W.C. and Netzer, N.C. (2009) Primary care: Is it the setting to address sleep disorders? Sleep Breath, 1, $1-9$.
[18] Chung, S.A., Jairam, S., Hussain, M.R. and Shapiro, C.M. (2001) Knowledge of sleep apnea in a sample grouping of primary care physicians. Sleep Breath, 5, 115-121. doi:10.1055/s-2001-17429

[19] Sassani, A., Findley, L.J., Kryger, M., Goldlust, E., George, C. and Davidson, T.M. (2004) Reducing motorvehicle collisions, costs, and fatalities by treating obstructive sleep apnea syndrome. Sleep, 27, 453-458.

[20] Tregear, S., Reston, J., Schoelles, K. and Phillips, B. (2010) Continuous positive airway pressure reduces risk of motor vehicle crash among drivers with obstructive sleep apnea: Systematic review and meta-analysis. Sleep, 33, 1373-1380.

[21] Yee, B., Campbell, A., Beasley, R. and Neill, A. (2002) Sleep disorders: A potential role in New Zealand motor vehicle accidents. Internal Medicine Journal, 32, 297304. doi:10.1046/j.1445-5994.2002.00229.x

[22] Antonopoulos, C.N., Sergentanis, T.N., Daskalopoulou, S.S. and Petridou, E.T. (2011) Nasal continuous positive airway pressure (nCPAP) treatment for obstructive sleep apnea, road traffic accidents and driving simulator performance: A meta-analysis. Sleep Medicine Reviews, 15, 301-310.

[23] Frey, W.C. and Pilcher, J. (2003) Obstrutive sleep-related breathing disorders in patients evaluated for bariatric surgery. Obesity Surgery, 13, 676-683. doi:10.1381/096089203322509228

[24] Biederman, J., Newcorn, I. and Sprich, S. (1991) Comorbidity of attention deficit hyperactivity disorder with conduct, depressive, anxiety, and other disorders. The American Journal of Psychiatry, 148, 564-577.

[25] Frick, P.J., Kamphaus, R.W., Lahey, B., Loeber, R., Christ, M.A.G. and Hart, E.L. (1991) Academic underachievement and the disruptive behavior disorders. Journal of Consulting and Clinical Psychology, 59, 289-294. doi:10.1037/0022-006X.59.2.289

[26] Barkley, R.A. (2006) Attention-deficit hyperactivity disorder: A handbook for diagnosis and treatment. The Guilford Press, New York.

[27] Cuccia, A.M., Campisi, G., Cannavale, R. and Colella, G. (2007) Obesity and craniofacial variables in subjects with obstructive sleep apnea syndrome: Comparisons of cephalometric values. Head \& Face Medicine, 3, 41. doi:10.1186/1746-160X-3-41

[28] Alkhali, M. and Lockey, R. (2011) Pediatric obstructive sleep apnea syndrome (OSAS) for the allergist. Annals of Allergy, Asthma \& Immunology, 107, 104-109. doi:10.1016/j.anai.2011.04.004

[29] Szabo, J. and Goldfarb, B. (2005) Metabolic syndrome risisng in adults and adolescents. DOC News, $2,7$.

[30] Southwell, C., Moallen, M. and Auckley, D. (2008) Cardiologists knowledge and attitudes about obstructive sleep apnea: A survey study. Sleep Breath, 12, 295-302. doi:10.1007/s11325-008-0170-1

[31] Olivarez, S.A., Ferres, M., Antony, K., Mattewal, A., Maheshwari, B., Sangi-Haghpeykar, H. and AagaardTillery, K. (2011) Obstructive sleep apnea screening in pregnancy, perinatal outcomes, and impact of maternal 
obesity. American Journal of Perinatology, 28, 651-658. doi:10.1055/s-0031-1276740

[32] Ohayon, M.M. (2003) The effects of breathing-related sleep disorders on mood disturbances in the general population. Journal of Clinical Psychiatry, 64, 11951200. doi:10.4088/JCP.v64n1009

[33] Yarlagadda, A., Kaushik, S. and Clayton, A.H. (2008) Obstructive sleep apnea, hypoxia, and metabolic syndrome in psychiatric and nonpsychiatric settings. Psychiatry, 5, 58-50.

[34] Yarlagadda, A., Brown, A.B. and Clayton, A.H. (2007) Onset of sleep apnea after initiation of psychotropic agents. Letter to the editor. Primary Care Companion to the Journal of Clinical Psychiatry, 9, 471

[35] Chung, F., Yegneswaran, B., Liao, P., Chung, S.A., Vairavanathan, S., Islam, S., Khajehdehi, A. and Shapiro, C.A. (2008) STOP Questionnaire. A tool to screen patients for obstructive sleep apnea. Anesthesiology, 108, 812-821. doi:10.1097/ALN.0b013e31816d91b5

[36] Drager, L.F., Polotsky, V.Y. and Lorenzi-Filho, G. (2011) Obstructive sleep apnea: An emerging risk factor for atherosclerosis. Chest, 140, 534-542. doi: $10.1378 /$ chest.10-2223

[37] Sert-Kuniyoshi, F.H., Squires, R.W., Korenfeld, Y.K., Somers, V.K., Pusalavidyasagar, S., Caples, S.M., John- son, L.L., Thomas, R.J. and Lopez-Jimenez, F. (2011) Screening for obstructive sleep apnea in early outpatient cardiac rehabilitation: Feasibility and results. Sleep Medicine, 12, 924-927. doi:10.1016/j.sleep.2010.11.014

[38] Lurie, A. (2011) Cardiovascular disorders associated with obstructive sleep apnea. Advances in Cardiology, 46, 197-266.

[39] Pronzato, C. (2010) Chronic obstructive pulmonary disease and obstructive sleep apnea. Association, consequences and treatment. Monaldi Archives for Chest Disease, 73, 155-161.

[40] Chen, Y.H., Kang, J.H., Lin, C.C., Wang, I.T., Keller, J.J. and Lin, H.C. (2011) Obstructive sleep apnea and the risk of adverse pregnancy outcomes. American Journal of Obstetrics \& Gynecology, 206, 136.e1-136.e5.

[41] Cooke, J.R., Ayalon, L., Palmer, B.W., Loredo, J.S., Corey-Bloom, J., Natarajan, L., Liu, L. and Ancoli-Israel, S. (2009) Sustained use of CPAP slows deterioration of cognition, sleep, and mood in patients with Alzheimer's disease and obstructive sleep apnea: A preliminary study. Journal of Clinical Sleep Medicine, 5, 305-309.

[42] Youssef, N.A., Ege, M., Angly, S.S., Strauss, J.L. and Marx, C.E. (2011) Is obstructive sleep apnea associated with ADHD? Annals of Clinical Psychiatry, 23, 213-224. 


\title{
Appendix
}

\author{
SLEEP APNEA SCALE ${ }^{\odot}$ \\ SELF RATING AND ASSESSMENT
}

Name:

Age:

Sex:

Date:

Education:

1) Do you wake up frequently, have trouble staying asleep or are restless at night? If you answered yes, for how long?
A. 1-6 Months
B. 6-12 Months
C. More Than 12 Months.

2) Do you miss work, are you late for appointments or social events or have you ever fallen asleep while driving/at traffic lights?

3) Do You Snore Or Does Your Partner Say You Stop Breathing For Short Periods While Asleep?

4) Do you suffer from allergies, asthma, or a history of nasal fractures, smoking or ADHD?

5) Do you feel sleep deprived, tired or sleepy upon waking up and during most of the day?

6) Do you currently take three or more prescription medications? If yes, List:
A
B.
C.

7) Do you consider yourself overweight? If so, circle by how much and what is your height?
A. 10-20 LBS
B. 20-40 LBS
C. 40 or More LBS. Height in inches

8) Do you suffer from headaches, chronic fatigue, depressed mood, decreased libido or memory problems?

9) Do you suffer from hypertension, heart attack, stroke, diabetes or dementia?

\begin{tabular}{|l|l|l|l|l|l|l|l|l|}
\hline $10 \%$ & $10 \%$ & $30 \%$ & $5 \%$ & $10 \%$ & $5 \%$ & $20 \%$ & $5 \%$ & $5 \%$ \\
\hline
\end{tabular}

Score of $50 \%$ or higher represents a high risk for obstructive sleep apnea. Give results to your doctor. You may need a referral for a sleep study.

Females with a history of child birth are at highter risk of OSA.

A Yarlagadda, S Kaushik, AH Clayton.

Disclaimer: the above results are for screening purposes only and do not necessarily constitute a medical diagnosis or treatment until you discuss them with your doctor in person.

Above scale represents the views of the authors and does not reflect the official policy or position of the Department of the Army, Department of Defense, or the United States government. 